\title{
A NOVEL RADAR-ABSORBING-MATERIAL \\ BASED ON EBG STRUCTURE
}

Qiang Gao,' Yan Yin, ${ }^{2}$ Dun-Bao Yan, ${ }^{1}$ and Nai-Chang Yuan ${ }^{1}$ 'Institute of Electronic Science and Engineering

National University of Defense Technology

Changsha, Hunan Province 410073, P. R. China

'Department of Applied Physics

National University of Defense Technology

Changsha, Hunan Province 410073, P. R. China

Received 24 April 2005

ABSTRACT: A novel ultra-thin radar-absorbing material (RAM) using metamaterials is presented and the absorption performance is examined. Due to the high-impedance property of the metamaterials, the thickness of the RAM is about several tenths of the center wavelength of the $a b$ sorption band, which is considerably thinner than conventional absorbers. The absorption bandwidth of the RAM is about several hundred megahertz. (1) 2005 Wiley Periodicals, Inc. Microwave Opt Technol Lett 47: 228-230, 2005; Published online in Wiley InterScience (www. interscience.wiley.com). DOI 10.1002/mop.21132

Key words: radar-absorbing-material (RAM); Salisbury screen; highimpedance-ground-plane (HIGP) 


\section{INTRODUCTION}

Metamaterials are a kind of artificial electromagnetic material that are typically realized by periodic metallization patterns arranged on dielectric substrates, and have electromagnetic characteristics which differ from the patterns, such as negative refraction $[1,2]$, electromagnetic band gap $[3,4]$, and high-impedance property $[4$, 5]. Due to their novel properties, metamaterials have a variety of applications from microwave to optical frequencies [1-5].

Of all the interesting properties, the high-impedance feature is of particular interest. As a potential application of the feature, N. Egheta has described the possibility of having thin absorbing screens using metamaterial surfaces [6]. D. J. Kern and D. H. Werner have designed ultra-thin absorbers by using high-impedance frequency-selective surfaces (HZ FSS) [7]. However, the patterns of HZ FSS designed by them using a genetic algorithm are rather complex and difficult to engineer.

In this paper, a novel ultra-thin radar-absorbing material (RAM) using the Sievenpiper high-impedance-ground-plane (HIGP) is presented. The new-style RAM takes the form of the Salisbury screen, which is a well-known kind of RAM, and the ultra-thin design is based on the high-impedance property of the HIGP.

\section{REFLECTION PROPERTY OF THE SALISBURY SCREEN}

First described in 1952 [8], the Salisbury screen radar absorber has been examined by many authors $[9,10]$. The simplest Salisburyscreen structure is composed of a thin lossy screen and a lossless spacer in front of a ground plane, as shown in Figure 1. The complex permeability $\mu$ and permittivity $\varepsilon$ of the lossy screen are both assumed to be homogeneous in the $X-Y$ plane and change in the $Z$-direction; thus, the normalized magnetic surface impedance $\alpha$ and electric surface admittance $\beta$ of the lossy screen are defined as

$$
\alpha=\frac{Z_{m}}{Z_{0}} \frac{j \omega \int_{0}^{\Delta} \mu(z) d z}{Z_{0}}, \beta \frac{\hat{Y}_{t}}{Y_{0}} \frac{j \omega \int_{0}^{\Delta} \varepsilon(z) d z}{Y_{0}},
$$

where $Z_{0}=\sqrt{\mu_{0} / \varepsilon_{0}}$ is the impedance of a vacuum, $Y_{0}=1 / Z_{0}$, and $\Delta$ is the depth of the lossy screen. The normalized admittance of the spacer is $Y_{s}$, and the depth is $d$.

In the vacuum (denoted as $n-2$ ) as well as the spacer (denoted as $n=1$ ), the electric field is the superposition of the incident wave and the reflective wave, $E_{n}=A_{n} e^{-j k_{n} d}+B_{n} e^{j k_{n} d}$,

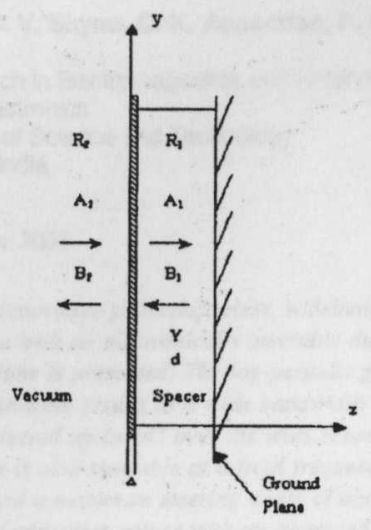

Flgure 1 Salisbury-screen geometry 


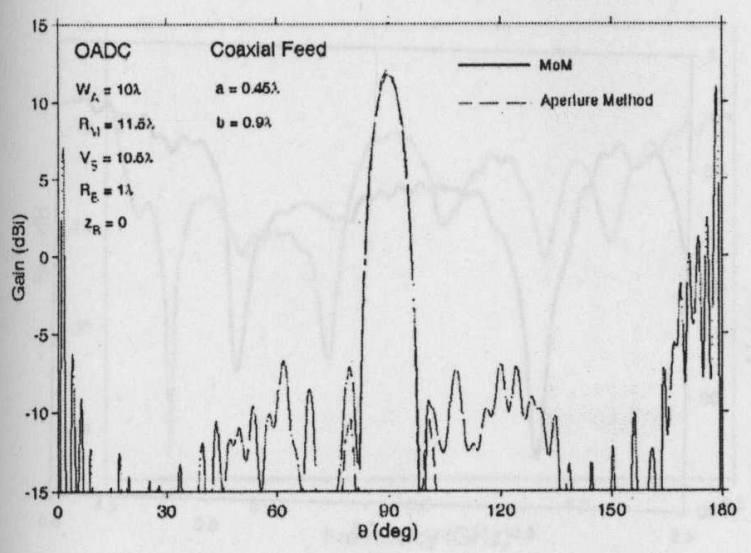

Figure 8 OADC radiation pattern evaluated by MoM (solid line) and aperture method (dashed line)

$$
\vec{E}_{A}= \pm V\left(\theta_{F}\right)\left|\frac{d_{2}-c_{2}+\left(d_{2}+c_{2}\right) \eta_{F}}{1+\eta_{F}^{2}}\right| \sqrt{\frac{\left|\eta_{F}\right|}{\left|f_{P}\left(1-e^{2}\right)\right|}} \hat{z}
$$

where the \pm sign refers to the $\mathrm{OADH}$ or $\mathrm{OADG}$ (OADE or $O A D C)$. The antenna far-field radiation is then obtained by integrating the $\mathrm{GO}$ aperture field (aperture method). The aperture stop is simply specified by $0 \leq\left|\theta_{F}\right| \leq\left|\theta_{E}\right|$, with the help of Eq. (13).

As the cylindrical aperture has a uniform phase distribution (according to GO), the far-field peak occurs at $\theta=90^{\circ}$. The corresponding directivity $D_{o}$ is compared against the maximum directivity $D_{\max }$ - given by Eq. (11) of [3] - of a uniform cylindrical aperture with the same dimensions and radiating the same feed power. The antenna efficiency is then defined by the ratio $D_{o} / D_{\text {max }}$, thus accounting for both illumination and spillover efficiencies. Figures 5 and 6 illustrate the antenna efficiencies obtained for mapping options I and II, respectively, as functions of $V_{S}$ and $R_{M}$ (still with $W_{A}=10 \lambda, R_{B}=1 \lambda$, and $z_{B}=0$ ). The efficiencies were obtained by the aperture method, with the help of Eqs. (11)-(14) with $a=0.45 \lambda$ and $b=0.9 \lambda$. As observed in those figures, the antenna efficiency strongly depends on $V_{s}$ and its maximum is obtained by compromising spillover losses (note that $\theta_{E}$ decreases as $V_{S}$ increases, increasing the subreflector spillover) and the uniform illumination of the aperture. Note that in Figures 5 and 6 the designs with maximum efficiencies correspond to $\mathrm{OADE}$ and $\mathrm{OADC}$ configurations, respectively.

From Figures 5 and 6 one can also observe that $R_{M}$ does not significantly affect the maximum efficiency; however, it does affect the antenna size. Table 1 compares the antenna volumes (defined by the cylinder that circumscribes the dual-reflector system) obtained for the designs with maximum efficiency, according to Figures 5 and 6 . As anticipated in section 2, the minimum volume occurs for $R_{M} \approx W_{A}$ and, consequently, $\beta \approx 45^{\circ}$ for both mapping options. For $\beta<45^{\circ}$ the volume approximately grows with $R_{M}^{2}$, although $V_{S}$ decreases. For $\beta>45^{\circ}$, the volume grows faster, as $R_{S}$ drastically increases while $R_{M}$ decreases.

To validate the previous procedure and show its usefulness, MoM analyses were conducted to evaluate of the radiation patterns of two representative antennas: an OADE with $R_{M}=12 \lambda$ and $V_{S}=8 \lambda$, and an OADC with $R_{M}=11.5 \lambda$ and $V_{S}=10.5 \lambda$, as depicted in Figures 7 and 8 , respectively. The main-beam characteristics yielded by the aperture method show good agreement with those of the MoM analyses. The aperture method predicts gains of 11.7 and $12 \mathrm{dBi}$ for the $\mathrm{OADE}$ and $\mathrm{OADC}$, respectively, while the 
MoM estimates 11.9 and $11.7 \mathrm{dBi}$, respectively. The small discrepancies between the different methods (about \pm 0.3 - $\mathrm{dB}$ in gain) is due to the strong electromagnetic coupling between the reflector surfaces of such compact arrangements.

\section{CONCLUSION}

This paper has considered the classical dual reflector for omnidirectional coverage using geometrical optics (GO) principles to obtain simple closed-form equations for the geometry design and for the vector field at the antenna cylindrical aperture. Main-beam radiation characteristics were estimated by integrating the GO aperture field (the aperture method). This procedure allows a parametric study to identify the geometrical and radiation features of different types of dual-reflector configurations. The OADE and $\mathrm{OADC}$ were chosen for compromising higher efficiencies and compactness. For these two configurations, the antenna performance yielded by the formulation presented in this work was compared against MoM analyses, showing good agreement for antennas with aperture widths $W_{A}=10 \lambda$ or larger.

\section{REFERENCES}

1. A.P. Norris and W.D. Waddoup, A millimetric wave omnidirectional antenna with prescribed elevation shaping, Proc $4^{\text {th }}$ Int Conf Antennas Propagat (ICAP), 1985, pp. 141-145.

2. M. Orefice and P. Pirinoli, Dual reflector antenna with narrow broadside beam for omnidirectional coverage, Electron Lett 29 (1993), 2158 2159.

3. A.G. Pino, A.M.A. Acuña, and J.O.R. Lopez, An omnidirectional dual-shaped reflector antenna, Microwave Opt Technol Lett 25 (2000), 371-374.

4. J.R. Bergmann, F.J.V. Hasselmann, and M.G.C. Branco, A singlereflector design for omnidirectional coverage, Microwave Opt Technol Lett 24 (2000), 426-429.

5. J.R. Bergmann and F.J.S. Moreira, An omnidirectional ADE reflector antenna, Microwave Opt Technol Lett 40 (2004), 250-254.

6. B.S. Westcott, F.A. Stevens and F. Brickell, GO synthesis of offset dual-reflecturs, IEE Proc $128 \mathrm{H}$ (1981), 11-18.

7. F.J.S. Moreira and A. Prata, Jr. Generalized classical axially symmetric dual-reflector antennas, IEEE Trans Antennas Propagat 49 (2001), 547-554. 